\section{SG-2: A promising lipolytic and pro-autophagic hit-compound to treat Alzheimer's disease}

\author{
Massimiliano Runfola, ${ }^{1}$ Michele Perni, ${ }^{2}$ \\ Simona Sestito, ${ }^{1}$ Grazia Chiellini, ${ }^{3}$ \\ Michele Vendruscolo, 2,4 \\ Simona Rapposelli ${ }^{1}$
}

1Department of Pharmacy, University of Pisa, Pisa, Italy; ${ }^{2}$ Department of Chemistry, University of Cambridge, Cambridge, United Kingdom; ${ }^{3}$ School of Medicine, University of Pisa, Pisa, Italy; 4 Interdepartmental Research Center in Biology and Pathology of Aging, University of Pisa, Italy

\begin{abstract}
The identification of efficient pharmacological tools for treatment of Alzheimer's disease (AD) represents one of the main challenges of our century. Due to the complex etiopathology and the several biological processes resulting impaired in $\mathrm{AD}$, the drug discovery process should focus on the development of new chemical entities able to target this multi-faceted impairment. We designed and synthetized a new analogue of 3-iodothyronamine, namely SG-2, which shares an interesting pleiotropic activity. Within this study, we explored SG-2 ability to promote beneficial effects in a C. Elegans model of $\mathrm{AD}$, using a novel technique developed at Cambridge University, which exploits an automated system of high-resolution cameras to evaluate in parallel the motility of a huge number of nematodes (up
\end{abstract}

to 5000 at time) in response to drug administration. Our results showed that SG-2 can promote lifespan and restores motility of worms back to the wildtype.

\section{Introduction}

Alzheimer's disease (AD) is a progressive pathological condition which affects multiple brain functions and several physiological pathways such as lipid and glucose metabolism, proteins phosphorylation and autophagic flux. This multi-faceted impairment leads to an aberrant protein aggregation and uncontrolled neuronal cell death, resulting in the well-known decline of cognitive functions. Today, there is a worldwide effort to find better ways to treat $A D$, delay its onset, and/or prevent it from developing. In this context, the improvement in up-to-date approaches and techniques to investigate new agents capable of interfering with $\mathrm{AD}$ progression still represents an urgent entail to be solved. Recently, we have designed and characterized a new class of synthetic small molecules bearing a biphenylmethane scaffold, namely SG compounds, to target the multi-faceted impairment which characterizes AD. ${ }^{1,2}$ Among them, SG-2 was identified as a promising hit-compound able to promote a rebalancing of autophagic flux, endowed of neuroprotective effects, and able to induce a metabolic reprogramming to favor lipid consumption. ${ }^{3}$
Correspondence: Simona Rapposelli, Department of Pharmacy, University of Pisa, Pisa, Italy.

E-mail: simona.rapposelli@unipi.it

Key words: Autophagy; neuroprotection; Alzheimer's Disease; lipolytic activity; C. Elegans.

Conference presentation: this paper was presented at the Second Centro 3R Annual Meeting - 3Rs in Italian Universities, 2019, June 20-21, University of Genoa, Italy.

Received for publication: 28 October 2019. Accepted for publication: 11 November 2019.

This work is licensed under a Creative Commons Attribution NonCommercial 4.0 License (CC BY-NC 4.0).

CCopyright: the Author(s), 2019

Licensee PAGEPress, Italy

Biomedical Science and Engineering 2019; 3(s3):121 doi:10.4081/bse.2019.121

\section{Materials and Methods}

To assess SG-2 potential in contrasting the progression of $\mathrm{AD}$ conditions, we tested it using a novel technique developed at Cambridge University which exploits an automated system of high-resolution cameras to evaluate in parallel the motility of a huge number of nematodes (up to 5000 at time) in response to drug administration. 4

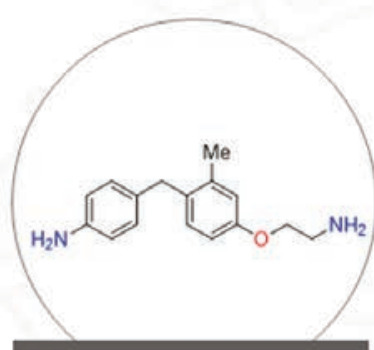

New Pleiotropic Agent SG2
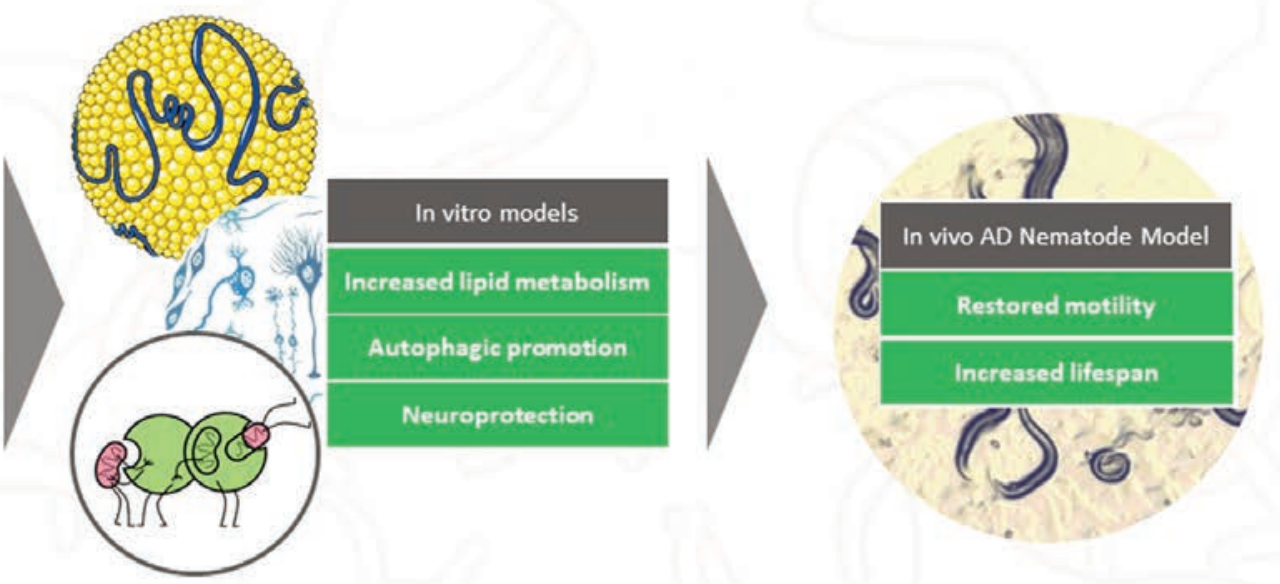

Figure 1. Effect of SG-2 in in vitro models and in vivo AD Neumatode model. 


\section{Results}

Our results showed that SG-2 can alter the decline of the morbidity of AD restoring nematode's motility back to the wild type when administrated to C. Elegans at a concentration of $1 \mu \mathrm{M}$. Moreover, we observed an enhancement of nematodes' lifespan when worms were treated with SG2 at the 4th day of life, i.e. when $\mathrm{A} \beta$ plaques are already formed. Surprisingly, no direct effect on $\mathrm{A} \beta$ formation has been observed in vitro. This result let us to speculate that the ability of SG2 to promote autophagy and induce lipid metabolism could represent a new strategy to delay or halt the progression of $\mathrm{AD}$.

\section{Conclusions}

We identified a novel lipolytic and proautophagic hit-compound able to promote beneficial effects in several AD models. Future studies are planned to outline the specific mechanism of action of this pleiotropic agent in order to validate the potential of SG2 as novel therapeutic tool for treatment of $\mathrm{AD}$.

\section{References}

1. Chiellini G, Nesi G, Digiacomo M, et al. Design, Synthesis, and Evaluation of
Thyronamine Analogues as Novel Potent Mouse Trace Amine Associated Receptor 1 (m TAAR1) Agonists. J Med Chem 2015;58:5096-107.

2. Chiellini G, Nesi G, Sestito S, et al. Hitto-lead optimization of mouse trace amine associated receptor 1 (mTAAR1) agonists with a diphenylmethane-scaffold: design, synthesis, and biological study. J Med Chem 2016;59:9825-36.

3. Bellusci L, Laurino A, Sabatini M, et al. New insights into the potential roles of 3-iodothyronamine (T1AM) and newly developed thyronamine-like TAAR1 agonists in neuroprotection. Front Pharmacol 2017;8:905. 